\title{
Bayesian inference for a partially observed birth-death process using data on proportions
}

\author{
R. J. Boys ${ }^{1 *}$ H. F. Ainsworth ${ }^{2}$ and C. S. Gillespie ${ }^{1}$ \\ ${ }^{1}$ School of Mathematics, Statistics \& Physics, Newcastle University, \\ Newcastle upon Tyne, NE1 7RU, UK. \\ ${ }^{2}$ Institute of Health and Society, Newcastle University, \\ Newcastle upon Tyne, NE1 4AX, UK.
}

\begin{abstract}
Stochastic kinetic models are often used to describe complex biological processes. Typically these models are analytically intractable and have unknown parameters which need to be estimated from observed data. Ideally we would have measurements on all interacting chemical species in the process, observed continuously in time. However, in practice, measurements are taken only at a relatively few time-points. In some situations, only very limited observation of the process is available, such as when experimenters can only observe noisy observations on the proportion of cells that are alive. This makes the inference task even more problematic. We consider a range of data-poor scenarios and investigate the performance of various computationally intensive Bayesian algorithms in determining the posterior distribution using data on proportions from a simple birth-death process.
\end{abstract}

Keywords: Partial observation; Gaussian process; sparse emulator.

\section{Introduction}

Biological modellers increasingly use stochastic kinetic models to describe the complex and stochastic nature of their experiments. Typically these models are analytically intractable and have unknown parameters which need to be estimated from observed data. Ideally the experiments would produce continuous-time measurements on all chemical species within the model, and with such data, Bayesian inference usually proceeds in a straightforward manner, often by taking conjugate priors for the model parameters. However, in practice, continuous-time measurements are not possible and measurements are taken only at a relatively few time-points. This complicates the inference as the observed data likelihood is typically intractable. Solutions to this problem often use data augmentation and thereby integrate over the unobserved continuous paths between observations; see, for example, Gibson and Renshaw [1998], Boys et al. [2008], Gibson and Renshaw [2001] and Golightly and Wilkinson [2005]. In some situations, experiments only yield a very limited view of

\footnotetext{
*email: richard.boys@ncl.ac.uk

Holly Ainsworth was supported by a PhD studentship from the UK Engineering and Physical Sciences Research Council. The authors would like to thank an Editor and three anonymous referees for comments that improved the paper.
} 
the underlying process. For example, all that may be observed could be noisy observations on the proportion of cells that are alive. This can make the inference task even more problematic. In this paper we consider a range of data-poor scenarios and build computationally intensive Bayesian algorithms to determine the posterior distribution. We will study a system in which measurements are available on the alive-status of individual cells and describe the (independent) dynamics of each cell using a simple birth-death process. Cell death is assumed to occur when its internal population (as described by the birth-death process) becomes extinct. The birth-death process provides useful toy model which captures a wide range of lifetime distributions for different choices of its birth and death rates and initial population size.

In section 2 , we describe the simple birth-death process and state some of the key analytic expressions that will be needed to formulate expressions for the likelihood function in the data scenarios we consider. Section 3 goes on to describe these data scenarios and outlines methods for obtaining the posterior distribution. In section 4 we consider the case where we do not have an analytic expression for the probability that the cell is dead at time $t$. Instead we have to base our inference scheme on simulated proportions of cell death obtained from running a simulator of the underlying (birth-death) process. In many cases, obtaining these simulated proportions within an MCMC scheme will be far too computer intensive and so we consider other methods which make use of Gaussian process emulators.

\section{The birth-death process}

The simple birth-death process is a well studied stochastic model. The model, which dates back to Yule [1925] and Feller [1939], has been widely used in biological applications; see, for example, its use as a model for the early stages of an epidemic in Kendall [1948]. Key useful attributes of the model are its simplicity and tractability. The model for population size $X$ in a typical cell can be written in chemical notation as $R_{1}: X \stackrel{\lambda}{\rightarrow} 2 X$ and $R_{2}: X \stackrel{\mu}{\rightarrow} \emptyset$, where $\lambda$ and $\mu$ are the birth and death rates (per member of the population).

The model is sufficiently simple that it is possible to obtain an analytic expression for its transition probabilities; see Renshaw [1993]. In particular, for $\lambda \neq \mu$ and an initial population of size $x_{0}$ within the cell, the probability of cell death (i.e. population extinction within the cell) in $[0, t]$ is

$$
P_{0}(t)=\left\{\frac{\mu-\mu e^{(\mu-\lambda) t}}{\lambda-\mu e^{(\mu-\lambda) t}}\right\}^{x_{0}}
$$

and the density of extinction by time $t$ is

$$
p_{0}(t)=\frac{x_{0} \mu^{x_{0}}(\lambda-\mu)^{2} e^{(\mu-\lambda) t}\left\{1-e^{(\mu-\lambda) t}\right\}^{x_{0}}}{\left\{1-e^{(\mu-\lambda) t}\right\}\left\{\lambda-\mu e^{(\mu-\lambda) t}\right\}^{\left(x_{0}+1\right)}} .
$$

Due to the tractable nature of the process, many authors have used the system as a test bed for different scenarios. For example, Dehay and Yao [2007] consider parameter inference when observing the process at discrete equi-distant time points, while Gillespie and Renshaw [2008] consider the case where only deaths are observed; see also the references therein. Inference for more general birth-death processes has also been considered by numerous authors; see, for example, Crawford and Suchard [2012] and Crawford et al. [2014]. Also Bladt and Sørensen [2005] consider inference in discretely observed Markov jump processes. 


\section{Inference under various data poor scenarios}

We now consider three observational scenarios and outline how realisations can be simulated from the parameter posterior distribution using MCMC methods. The scenarios are

(a) the times of cell death are known (exactly), with data $\boldsymbol{t}^{e}=\left(t_{1}^{e}, t_{2}^{e}, \ldots, t_{m}^{e}\right)$;

(b) the status of each cell is observed at time points $t_{1}, t_{2}, \ldots, t_{B}$, leading to data $\boldsymbol{n}=\left(n_{1}, n_{2}, \ldots, n_{B}, n_{B+1}\right)$, where $n_{i}$ is the number of cells that die in time bin $\left(t_{i-1}, t_{i}\right]$, with $\sum_{i} n_{i}=m, t_{0}=0$ and $t_{B+1}=\infty$;

(c) only noisy measurements of the proportion of dead cells (out of a large number of cells) are available at time points $t_{1}, t_{2}, \ldots, t_{B}$, giving $\boldsymbol{p}^{\text {obs }}=\left(p_{1}^{\text {obs }}, p_{2}^{\text {obs }}, \ldots, p_{B}^{\text {obs }}\right)$, where $p_{i}^{o b s}$ is the observed proportion of dead cells at time $t_{i}$.

To simplify what follows, we will assume that the initial population level in each cell is known to be $x_{0}=10$. Note that the methods we describe in this section make use of the analytic expressions (1) and (2).

\subsection{Inference using known death times}

If the times of each cell death are known, the likelihood is $\pi\left(\boldsymbol{t}^{e} \mid \lambda, \mu\right)=\prod_{i=1}^{m} p_{0}\left(t_{i}^{e} \mid \lambda, \mu\right)$, where $p_{0}(\cdot)$ is as in (2). Therefore, by Bayes Theorem, the posterior density is given by

$$
\pi\left(\lambda, \mu \mid \boldsymbol{t}^{e}\right) \propto \pi(\lambda, \mu) \pi\left(\boldsymbol{t}^{e} \mid \lambda, \mu\right)
$$

where $\pi(\lambda, \mu)$ is the prior density for $(\lambda, \mu)$. This posterior distribution is non-standard but can be targeted using a simple Metropolis-Hastings scheme which uses a joint update consisting of (independent) random walks (on a log scale) for each parameter. Such a scheme accepts proposal $\left(\lambda^{*}, \mu^{*}\right)$ with acceptance probability $\min (1, A)$, where

$$
A=\frac{\lambda^{*} \mu^{*} \pi\left(\lambda^{*}, \mu^{*}\right)}{\lambda \mu \pi(\lambda, \mu)} \times \prod_{i=1}^{m} \frac{p_{0}\left(t_{i}^{e} \mid \lambda^{*}, \mu^{*}\right)}{p_{0}\left(t_{i}^{e} \mid \lambda, \mu\right)} .
$$

Here the additional term $\lambda^{*} \mu^{*} /(\lambda \mu)$ results from the log-normal proposal ratio $q\left(\lambda, \mu \mid \lambda^{*}, \mu^{*}\right) / q\left(\lambda^{*}, \mu^{*} \mid \lambda, \mu\right)$.

\subsection{Inference using cell census data}

Now suppose that the exact cell death times are not observed and instead only the dead-alive status of each cell is observed at a series of census times $t_{1}, t_{2}, \ldots, t_{B}$. From this information we can determine the number of cells $n_{i}$ that die in $\left(t_{i-1}, t_{i}\right], i=1, \ldots, B+1$. The likelihood is now $\pi(\boldsymbol{n} \mid \boldsymbol{t}, \lambda, \mu)=\prod_{i=1}^{B+1}\left\{P_{0}\left(t_{i} \mid \lambda, \mu\right)-P_{0}\left(t_{i-1} \mid \lambda, \mu\right)\right\}^{n_{i}}$, where $t_{0} \equiv 0$ and $P_{0}(\cdot)$ is as in (1). As before, realisations can be simulated from the posterior distribution, now given by

$$
\pi(\lambda, \mu \mid \boldsymbol{n}, \boldsymbol{t}) \propto \pi(\lambda, \mu) \pi(\boldsymbol{n} \mid \boldsymbol{t}, \lambda, \mu),
$$

using joint independent random walk proposals (on a log scale). Here proposals $\left(\lambda^{*}, \mu^{*}\right)$ are accepted with probability $\min (1, A)$, where

$$
A=\frac{\lambda^{*} \mu^{*} \pi\left(\lambda^{*}, \mu^{*}\right)}{\lambda \mu \pi(\lambda, \mu)} \times \prod_{i=1}^{B+1}\left\{\frac{P_{0}\left(t_{i} \mid \lambda^{*}, \mu^{*}\right)-P_{0}\left(t_{i-1} \mid \lambda^{*}, \mu^{*}\right)}{P_{0}\left(t_{i} \mid \lambda, \mu\right)-P_{0}\left(t_{i-1} \mid \lambda, \mu\right)}\right\}^{n_{i}}
$$




\subsection{Inference using noisy measurements of cell death proportions}

A more typical experimental scenario is one where we cannot observe the numbers of cells that die between the census time points. Instead all that can be observed is the proportion of cells that are dead, and this measurement is also subject to error. We will assume an additive normal error structure on the logit scale, that is, the observation model is

$$
y_{i}=\operatorname{logit} p_{t_{i}}(\lambda, \mu)+\sigma \varepsilon_{i},
$$

for $i=1,2, \ldots, B$, where $y_{i}=\operatorname{logit} p_{i}^{o b s}$ is the logit of the observed proportion, $p_{t}(\lambda, \mu)$ is the probability of a cell being dead at time $t$ and the $\varepsilon_{i}$ are independent standard normal quantites. Note that, for this simple birth-death process, we have a closed form expression (1) for $p_{t}(\lambda, \mu)$. We will assume that $(\lambda, \mu)$ and $\sigma$ are independent a priori, in which case the posterior density is given by

$$
\pi(\lambda, \mu, \sigma \mid \boldsymbol{y}) \propto \pi(\lambda, \mu) \pi(\sigma) \pi(\boldsymbol{y} \mid \lambda, \mu, \sigma) .
$$

In this scenario, the likelihood is

$$
\pi(\boldsymbol{y} \mid \lambda, \mu, \sigma)=\prod_{i=1}^{B} \phi\left\{y_{i} \mid \operatorname{logit} p_{t_{i}}(\lambda, \mu), \sigma^{2}\right\}
$$

where $\phi(\cdot \mid m, v)$ denotes a normal density with mean $m$ and variance $v$. We can build an MCMC scheme targeting the posterior distribution by a joint Metropolis-Hastings step with independent symmetric normal random walk proposals (on the $\log$ scale) for $\lambda, \mu$ and $\sigma$. A proposal $\left(\lambda^{*}, \mu^{*}, \sigma^{*}\right)$ is accepted with probability $\min (1, A)$, where

$$
A=\frac{\lambda^{*} \mu^{*} \sigma^{*} \pi\left(\lambda^{*}, \mu^{*}\right) \pi\left(\sigma^{*}\right)}{\lambda \mu \sigma \pi(\lambda, \mu) \pi(\sigma)} \times \prod_{i=1}^{B} \frac{\phi\left\{y_{i} \mid \operatorname{logit} p_{t_{i}}\left(\lambda^{*}, \mu^{*}\right), \sigma^{* 2}\right\}}{\phi\left\{y_{i} \mid \operatorname{logit} p_{t_{i}}(\lambda, \mu), \sigma^{2}\right\}} .
$$

\subsection{Comparison of data scenarios}

We will compare the posterior distributions under these three data scenarios by using simulated datasets. We will simulate the cell dynamics assuming that each cell has an initial population size $x_{0}=10$ and take the birth and death rates as $\lambda=0.6$ and $\mu=1$. In our analyses we assume that the prior distribution is not inconsistent with the truth by taking fairly weak independent log-normal components, with each component median set at the true value, that is, take $\lambda \sim \operatorname{LN}(\log 0.6,2)$ and $\mu \sim \operatorname{LN}(0,2)$.

We base our analysis on datasets of size $n=100$ and $n=1000$ simulated under scenario (a). In scenario (b), the simulated data from scenario (a) is discretised and we take the final bin to be $\left(t_{B}=11, t_{B+1}=\infty\right)$ so that the final bin contains all cells which die after time $t=11$. We consider the effect of different discretisations of the datasets by fixing the census times in $(1,11]$ to be on regular grids of different size. Note that we do not consider early time-points in $(0,1)$ as, with our choice of parameters $(\lambda, \mu)$, the process changes very little in this time interval. Specifically we consider pooling the data into $B=10,25,50$ intervals, that is, look at time bins (except the final bin) with width $t_{i}-t_{i-1}=10 / B=1,0.4,0.2, i=1, \ldots, B$. We also investigate in scenario (c), the impact on the posterior distribution of only observing cell death proportions at census times. Here we simulate datasets on proportions with small, medium and large levels of measurement error $(\sigma=0.3,0.5,0.7)$. In the analysis of these datasets we take $\sigma \sim \operatorname{LN}(\log 0.5,0.5)$ as another independent component in our prior distribution.

We now examine the effect of these various data scenarios on posterior inference. In all cases, the posterior distribution has been constructed after running MCMC schemes in which the first 100 

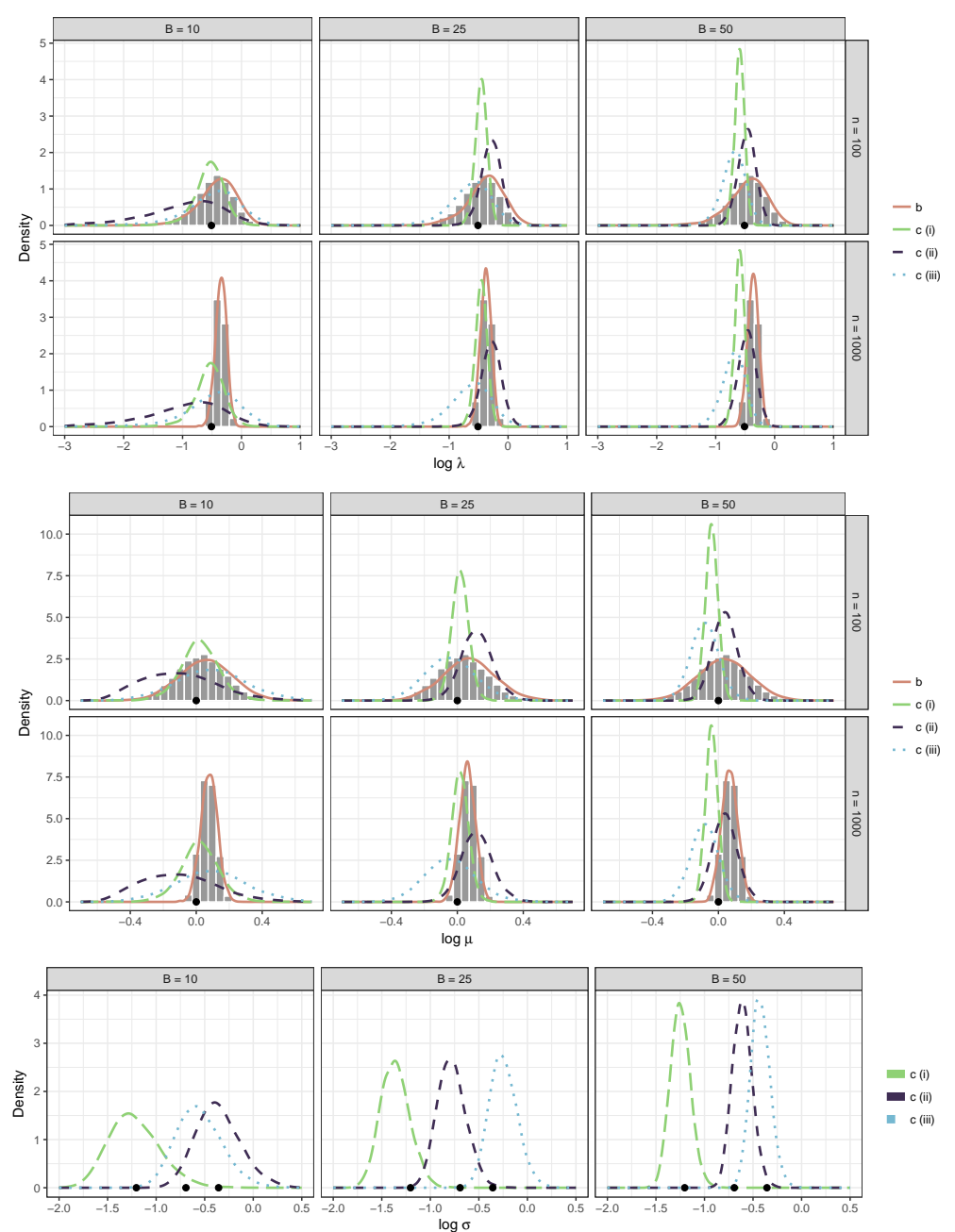

Figure 1: Marginal posterior density histograms for $\log \lambda$ (top) and $\log \mu$ (middle) using the exact algorithm and noise-free data from scenario (a), together with posterior densities for $\log \lambda$, $\log \mu$ and $\log \sigma$ (bottom) using noise-free data from scenario (b) and noisy data from scenario (c), with different sizes, levels of discretisation $(B)$ and levels of measurement error $(\sigma)$. Posterior densities determined by using $10^{3}$ realisations from MCMC schemes. The true values are given by solid circles.

iterations have been discarded as burnin and then the next $10^{6}$ realisations thinned by $10^{3}$ to obtain an (almost un-autocorrelated) posterior sample of size $10^{3}$. Figure 1 shows the marginal posterior densities for model parameters $\log \lambda, \log \mu$ and $\log \sigma$ under scenarios (a), (b) and (c) described above. It is clear that, under scenarios (a) and (b), the level of discretisation in the data has very little effect on the posterior distribution, even for the most coarse discretisation $(B=10)$. In general, the marginal posterior distributions under scenario (c) have greater precision than those under scenarios (a) and (b). Finally, and unsurprisingly, under scenario (c), posterior uncertainty for the model parameters increases as the level of noise $(\sigma)$ increases in the data.

\section{Inference using simulator output from the underlying process}

In the previous section, determining the posterior distribution using noisy data on proportions (scenario (c)) was made rather straightforward because we have an analytic expression for the 
probability of cell death $p_{t}(\lambda, \mu)$. Unfortunately this is generally not the case for stochastic kinetic models. Therefore we now explore the impact of using alternative inference strategies which rely instead on using simulated realisations from the model.

We can estimate $p_{t}(\lambda, \mu)$ by first simulating cell trajectories for each of $n$ cells and then calculating the proportion of these cells that are dead at time $t$. Such an estimate $\widehat{p}_{t, n}(\lambda, \mu)$ has binomial sampling error since $n \widehat{p}_{t, n}(\lambda, \mu) \sim \operatorname{Bin}\left\{n, p_{t}(\lambda, \mu)\right\}$, and is unbiased and consistent. As before we can build an MCMC scheme by using a Metropolis-Hastings step with independent symmetric normal random walk proposals (on the $\log$ scale) for $\lambda, \mu$ and $\sigma$. After simulating from the model to obtain a path of proportions $\widehat{\boldsymbol{p}}_{n}^{*}=\left\{\widehat{p}_{t_{i}, n}\left(\lambda^{*}, \mu^{*}\right), i=1, \ldots, B\right\}$ for some choice of $n$, we can then accept the proposal with probability $\min (1, A)$, where

$$
A=\frac{\pi\left(\lambda^{*}\right) \pi\left(\mu^{*}\right)}{\pi(\lambda) \pi(\mu)} \times \prod_{i=1}^{B} \frac{\phi\left(y_{i} \mid \text { elogit } \widehat{p}_{t_{i}, n}^{*}, \sigma^{2}\right)}{\phi\left(y_{i} \mid \text { elogit } \widehat{p}_{t_{i}, n}, \sigma^{2}\right)} \times \frac{\lambda^{*} \mu^{*}}{\lambda \mu}
$$

and

$$
\text { elogit } \widehat{p}=\log \left(\frac{\widehat{p}+0.5 / n}{1-\widehat{p}+0.5 / n}\right)
$$

is the empirical logit. We use the empirical logit here as this removes any problems with pathological cases $(\widehat{p}=0$ or 1$)$. Note that, for finite $n$, this scheme does not target the exact posterior distribution but it does so asymptotically as the proportion estimate is consistent and the likelihood terms are based on the asymptotic sampling distribution of the empirical logit.

The above scheme will work well if $n$ is very large but, in practice, limited computing resources will result in $n$ being sufficiently small that account needs to be taken of the sampling variation in these estimated proportions. For large $n$, the sampling distribution of the empirical logit (elogit $\widehat{p}$ ) is a normal distribution with mean logit $p$ and variance $1 /\{n \widehat{p}(1-\widehat{p})\}$. Thus, taking an improper constant prior for logit $p$ gives its posterior distribution as a normal distribution with mean elogit $\widehat{p}$ and variance $1 /\{n \widehat{p}(1-\widehat{p})\}$. Therefore we can integrate out posterior uncertainty about logit $p$ in the observation model, modifying the likelihood to

$$
\pi(\boldsymbol{y} \mid \lambda, \mu, \sigma)=\prod_{i=1}^{B} \phi\left(y_{t_{i}} \mid \text { elogit } \widehat{p}_{t_{i}, n}, \sigma^{2}+1 /\left\{n \widehat{p}_{t_{i}, n}\left(1-\widehat{p}_{t_{i}, n}\right)\right\}\right)
$$

with consequent changes to the MCMC acceptance probability.

Incidentally, it is possible to construct a pseudo-marginal particle filter to target the posterior $\pi(\lambda, \mu, \sigma \mid \boldsymbol{y})$ exactly; see, for example, Andrieu et al. [2010]. We looked at schemes that use either a Monte Carlo or a sequential Monte Carlo estimate of the likelihood $\pi(\boldsymbol{y} \mid \lambda, \mu, \sigma)$. However we found that, for our simple birth-death process, these schemes suffered from a much inferior computational performance (effective sample size per cpu second) than the scheme outlined above. This however might not be the case in larger more complex models.

\subsection{Gaussian process emulators}

The previous analysis required that proportions of cell death be simulated at each step of the MCMC algorithm. In all but the most simple models, simulating from the underlying model to obtain these proportions is far too time consuming. For example, calculating 100 proportions, each from $n=1000$ realisations over $(0,10)$ of the birth-death model, takes around $1 \mathrm{cpu}$ sec whereas it takes around $700 \mathrm{cpu}$ secs to generate the same information from the slightly larger Schlögl model [Owen et al., 2015], and much longer for more complex models. In this section we consider how Gaussian process (GP) emulators might be used to expedite inference when the simulator is not very quick; see, for example, Rasmussen and Williams [2006] for a background on GP emulators. They 
have been used by many authors for the emulation of complex deterministic models [Kennedy and O'Hagan, 2000, 2001] and for complex stochastic models [Henderson et al., 2009, 2010, Baggaley et al., 2012].

Determining an estimate $\widehat{p}_{t, n}(\lambda, \mu)$ of the cell death proportion at time $t$ is too computer-intensive and so we seek to model its sampling distribution, smoothing over $(\lambda, \mu)$-values and accounting for binomial sampling error, using a Gaussian process. We know that, for large $n$, elogit $\widehat{p}_{t, n}$ is almost normally distributed and so we will seek a Gaussian process emulator (approximation) for $x_{t, n}(\lambda, \mu)=$ elogit $\widehat{p}_{t, n}(\lambda, \mu)$. Note that, because of the form of the likelihood, we do not need an emulator across time. Rather, we need an emulator only at the time points at which data are observed. Thus we will need to construct $B$ GP emulators over $(\lambda, \mu)$-space. The process of fitting each GP emulator is fairly straightforward and a major computational benefit is that they can be fitted in parallel.

We now describe how a GP emulator can be constructed for a particular time point $t$. The inputs to the GP are $\boldsymbol{\theta}=(\lambda, \mu)$. First we need to construct our training data, that is, determine the value of $x_{t, n}(\boldsymbol{\theta})$ at a number of $\boldsymbol{\theta}$-values. There are many possible choices of $\boldsymbol{\theta}$-values to use: we will use a maximin Latin hypercube design (LHD) as these are space filling and have been shown to be effective in other work [Henderson et al., 2009, Baggaley et al., 2012]. We begin by constructing an $n_{d}=2000$-point LHD in $(\log \lambda, \log \mu)$ over the central $95 \%$ region of the prior distribution and then exclude any design points that give extreme proportions, that is, proportions that are clearly inconsistent with the data; here we exclude proportions outside $(0.005,0.995)$. The main reason for this pragmatic step is that when using relatively small designs, design points which have extreme (logit) proportions can be very influential in the GP fit and lead to GPs which fit poorly in the main area of posterior support. The limits of the interval $(0.005,0.995)$ we report here were determined by sequentially expanding the range from $(0.05,0.95)$ until a significant change to the GP fit was observed. After accounting for such deletions, this typically left around $n_{d}=150$ design points to use to fit the GPs.

The benefit of using a Gaussian process emulator for $x_{t, n}(\boldsymbol{\theta})$ is that, as the distribution of $x_{t, n}(\boldsymbol{\theta})$ at any finite collection of points $\boldsymbol{\Theta}=\left(\boldsymbol{\theta}_{i}, i=1, \ldots, n_{d}\right)$ has a Gaussian distribution, the fitted GP has a Gaussian distribution for $x_{t, n}\left(\boldsymbol{\theta}^{*}\right)$ at a new point $\boldsymbol{\theta}^{*}$. This distribution has mean and variance that depend on the prior mean function $m_{t}(\boldsymbol{\theta})$ and covariance function $K_{t}\left(\boldsymbol{\theta}, \boldsymbol{\theta}^{\prime}\right)$ of the GP and the training data $\mathcal{D}_{t}=\left\{\left(\boldsymbol{\theta}_{i}, x_{t, n}\left(\boldsymbol{\theta}_{i}\right)\right), i=1, \ldots, n_{p}\right\}$. We can also account for the training data being estimated proportions by adding a nugget term to the covariance function. Thus $x_{t, n}\left(\boldsymbol{\theta}^{*}\right) \mid \mathcal{D}_{t} \sim N\left\{m_{t}^{*}\left(\boldsymbol{\theta}^{*}\right), v_{t}^{*}\left(\boldsymbol{\theta}^{*}\right)\right\}$, where

$$
m_{t}^{*}\left(\boldsymbol{\theta}^{*}\right)=m_{t}\left(\boldsymbol{\theta}^{*}\right)+\tilde{K}_{t}\left(\boldsymbol{\theta}^{*}, \boldsymbol{\Theta}\right)^{\top} \tilde{K}_{t}(\boldsymbol{\Theta}, \boldsymbol{\Theta})^{-1}\left\{\boldsymbol{y}_{t}-\boldsymbol{m}_{t}(\boldsymbol{\Theta})\right\}
$$

and

$$
v_{t}^{*}\left(\boldsymbol{\theta}^{*}\right)=\tilde{K}_{t}\left(\boldsymbol{\theta}^{*}, \boldsymbol{\theta}^{*}\right)-\tilde{K}_{t}\left(\boldsymbol{\theta}^{*}, \boldsymbol{\Theta}\right)^{\top} \tilde{K}_{t}(\boldsymbol{\Theta}, \boldsymbol{\Theta})^{-1} \tilde{K}_{t}\left(\boldsymbol{\Theta}, \boldsymbol{\theta}^{*}\right)
$$

where

$$
\tilde{K}_{t}(\boldsymbol{\Theta}, \boldsymbol{\Theta})=K_{t}(\boldsymbol{\Theta}, \boldsymbol{\Theta})+\operatorname{diag}\left(n \operatorname{eexpit}\left\{m_{t}^{*}\left(\boldsymbol{\theta}_{i}\right)\right\}\left[1-\operatorname{eexpit}\left\{m_{t}^{*}\left(\boldsymbol{\theta}_{i}\right)\right\}\right]\right)^{-1}
$$

and eexpit is the inverse of the empirical logit, that is, eexpit $(m)=\left\{e^{m}(1+0.5 / n)-0.5 / n\right\} /\left(1+e^{m}\right)$.

Inspection of the training data $\mathcal{D}_{t}$ shows that a mean function which includes linear and quadratic terms in $\log \lambda$ and $\log \mu$ will capture most of the dependence on the inputs. We chose to estimate the parameters in this function using least squares. An alternative might be to take a fully Bayesian approach and perhaps assign weak prior information to these parameters. However, as the number of training points $n_{d}$ is reasonably large, this fully Bayesian approach typically results in the fitted 
process being a Student- $t$ process with a large number of degrees of freedom; see Shah et al. [2014]. This is a more complicated process but one which is very close to a Gaussian process. We therefore choose to ignore posterior uncertainty in the parameters of the mean function and use the more straightforward Gaussian process. Note that this (simple) approach essentially fits a zero mean Gaussian process to the residuals from the least squares fit. Thus we take mean function

$$
m_{t}(\boldsymbol{\theta})=b_{0 t}+b_{1 t} \log \lambda+b_{2 t} \log \mu+b_{3 t}(\log \lambda)^{2}+b_{4 t}(\log \mu)^{2}+b_{5 t}(\log \lambda)(\log \mu),
$$

where the $b_{i t}$ are the least squares estimates. We use a Gaussian covariance function

$$
K_{t}\left(\boldsymbol{\theta}_{i}, \boldsymbol{\theta}_{j} \mid a_{t}, \boldsymbol{r}_{t}\right)=a_{t} \exp \left\{-\frac{\left(\log \lambda_{i}-\log \lambda_{j}\right)^{2}}{r_{1 t}^{2}}-\frac{\left(\log \mu_{i}-\log \mu_{j}\right)^{2}}{r_{2 t}^{2}}\right\}
$$

which has a variance parameter $a_{t}$ and correlation length parameters $\boldsymbol{r}_{t}=\left(r_{1 t}, r_{2 t}\right)$ and we assign fairly weak independent $\log$-normal $\mathrm{LN}(0,10)$ priors to these parameters. Their posterior density is given by

$$
\pi\left(a_{t}, \boldsymbol{r}_{t} \mid \mathcal{D}_{t}\right) \propto \pi\left(a_{t}, \boldsymbol{r}_{t}\right) \pi\left(\mathcal{D}_{t} \mid a_{t}, \boldsymbol{r}_{t}\right),
$$

where the likelihood term $\pi\left(\mathcal{D}_{t} \mid a_{t}, \boldsymbol{r}_{t}\right)$ is an $n_{d}$-dimensional normal density with mean $m_{t}(\boldsymbol{\Theta})$ and covariance matrix $\tilde{K}_{t}(\boldsymbol{\Theta}, \boldsymbol{\Theta})$. Realisations from this posterior can be obtained via a MetropolisHastings algorithm with (independent) symmetric random walk proposals (on the log scale). Strictly speaking the fitted GP should be $E\left\{x_{t, n}\left(\boldsymbol{\theta}^{*}\right) \mid \mathcal{D}_{t}\right\}$, where the expectation is taken with respect to the posterior distribution of the GP parameters $\left(a_{t}, \boldsymbol{r}_{t}\right)$. However, like many authors, we found very little difference between this fitted GP and its delta approximation, that is, the fitted GP evaluated at the posterior mean of its parameters [Henderson et al., 2009, Baggaley et al., 2012].

Recall that the benefit of using a Gaussian process emulator for $x_{t, n}(\lambda, \mu)$ is that the fitted GP at a new point $\left(\lambda^{*}, \mu^{*}\right)$ has a Gaussian distribution, with $x_{t, n}\left(\lambda^{*}, \mu^{*}\right) \mid \mathcal{D}_{t} \sim N\left\{m_{t}^{*}\left(\lambda^{*}, \mu^{*}\right), v_{t}^{*}\left(\lambda^{*}, \mu^{*}\right)\right\}$, where the mean and variance terms are as in (4) and (5). We can now use these fitted GPs to approximate the distribution of the observed proportions and thereby approximate the likelihood (3) as

$$
\left.\pi(\boldsymbol{y} \mid \lambda, \mu, \sigma)=\prod_{i=1}^{B} \phi\left(y_{t_{i}} \mid m_{t_{i}}^{*}(\lambda, \mu), v_{t_{i}}^{*}(\lambda, \mu)+\sigma^{2}\right\}\right) .
$$

Therefore we can obtain a posterior sample via a Metropolis-Hastings algorithm which uses (independent) symmetric random walks (on a log scale) for each parameter. Note that this algorithm is very fast compared to the previous one as there is no need to simulate realisations from the model this is the benefit of using GP emulators which have been fitted off-line.

\subsubsection{Emulators with sparse covariance functions}

Computational efficiency gains may be achieved if the emulators are constructed using a sparse covariance function. The idea is to take advantage of the near sparsity of covariance matrices used in GP calculations by constructing them in a way such that they can be stored as sparse matrices. Here the main gain is that computationally efficient sparse matrix algorithms can then be used to speed up operations such as matrix inversions which would otherwise scale with $\mathcal{O}\left(n_{d}^{3}\right)$, where $n_{d}$ is the number of points in GP design $\boldsymbol{\Theta}$. This speed-up can be particularly beneficial when fitting GP as such matrix inversions are required at each step of the MCMC fitting algorithm. Also the loss in accuracy of the GP is small when the covariance matrices used to fit the GP are nearly sparse. 
Kaufman et al. [2011] describe a sparse covariance function for a process with input dimension $n_{p}$ : it has $(i, j)$ th entry

$$
K\left(\boldsymbol{\theta}, \boldsymbol{\theta}^{\prime}\right)_{i j}=a \prod_{k=1}^{n_{p}} R_{k}\left(\Delta_{i j k} ; \tau_{k}\right)
$$

where $\Delta_{i j k}=\left|\theta_{i k}-\theta_{j k}^{\prime}\right|$ and the correlation function in dimension $k$ is the Bohman function

$$
R_{k}\left(\Delta_{i j k} ; \tau_{k}\right)= \begin{cases}\left(1-\Delta_{i j k} / \tau_{k}\right) \cos \left(\pi \Delta_{i j k} / \tau_{k}\right)+\sin \left(\pi \Delta_{i j k} / \tau_{k}\right) / \pi, & \Delta_{i j k}<\tau_{k} \\ 0, & \text { otherwise. }\end{cases}
$$

These functions typically look like a squared exponential function but with the decay truncated at distance $\tau_{k}$. Essentially each hyperparameter $\tau_{k}$ measures the distance between two inputs in dimension $k$ before the output is assumed to be uncorrelated. The algorithm begins by scaling all inputs to lie between 0 and 1 , so that the $\tau_{k} \in(0,1)$. The level of sparsity $s$ to be imposed on the covariance function is specified by the user and represents a trade-off between computational efficiency and accuracy. The level of sparsity is induced through the prior distribution for $\boldsymbol{\tau}=\left(\tau_{1}, \ldots, \tau_{n_{p}}\right)$. This is taken to be a uniform distribution over $\left\{\boldsymbol{\tau} \in(0,1)^{n_{p}}: \sum_{k=1}^{n_{p}} \tau_{k} / n_{p} \leq c\right\}$, where $c$ is chosen to satisfy $c(2-c)=(1-s)^{1 / n_{p}}$. Therefore, for example, taking the sparsity level $s=0.90$ will ensure that $90 \%$ of the off-diagonal elements of $K\left(\boldsymbol{\theta}, \boldsymbol{\theta}^{\prime}\right)$ are zero. Taking $s=0.90$ in our two dimensional problem gives a uniform prior over the triangle $\left\{\boldsymbol{\tau} \in(0,1)^{2}: \tau_{1}+\tau_{2} \leq 2 c=0.346\right\}$.

Using a procedure very similar to that used to fit the non-sparse emulator at time $t$, we determine the posterior distribution for the hyperparameters $\left(a_{t}, \boldsymbol{\tau}_{t}\right)$ by first taking the prior distribution to have independent components, with the above prior for $\tau_{t}$ and $a_{t} \sim \operatorname{LN}(0,10)$, and then fitting the GP via a suitable MCMC scheme. As with the non-sparse emulators, the fitted sparse GPs ignore posterior uncertainty on the hyperparameters and simply fix them at their posterior mean.

\subsection{Effect of using non-sparse and sparse emulators on the posterior distribution}

We now look at the effect of using non-sparse and sparse emulators on posterior inference for our model parameters. Here we compare the marginal posterior distributions obtained using four different inference schemes:

(a) $p_{t}(\lambda, \mu)$ is known (labelled 'exact' in the figure)

(b) $p_{t}(\lambda, \mu)$ is unknown and is estimated using the simulator (simulator)

(c) $p_{t}(\lambda, \mu)$ is unknown and is estimated using a non-sparse emulator (emulator)

(d) $p_{t}(\lambda, \mu)$ is unknown and is estimated using a sparse emulator (sparse), with sparsity level $s=0.90$.

As in the previous section, data have been simulated from the birth-death process, here using parameter values $\lambda=0.6, \mu=1$ and $\sigma=0.5$, and an initial population size $x_{0}=10$. Also, as before, the prior distribution has independent components, with $\lambda \sim \operatorname{LN}(\log 0.6,2), \mu \sim \operatorname{LN}(0,2)$ and $\sigma \sim \mathrm{LN}(\log 0.5,0.5)$.

In all cases, the posterior distribution has been constructed after running MCMC schemes in which the first $10^{3}$ iterations have been discarded as burnin and then the next $10^{4}$ realisations thinned by 10 to obtain an (almost un-autocorrelated) posterior sample of size $10^{3}$. Figure 2 shows the marginal posterior densities for $\log \lambda$ (top), $\log \mu$ (middle) and $\log \sigma$ (bottom). Within each panel, columns show different levels of data discretisation $(B)$ and rows show the number of simulations $(n)$ used to generate approximate proportions $\widehat{p}_{t, n}$. The figure clearly shows that regardless of inference method, the (approximate) posterior distribution is located in very similar regions of parameter 

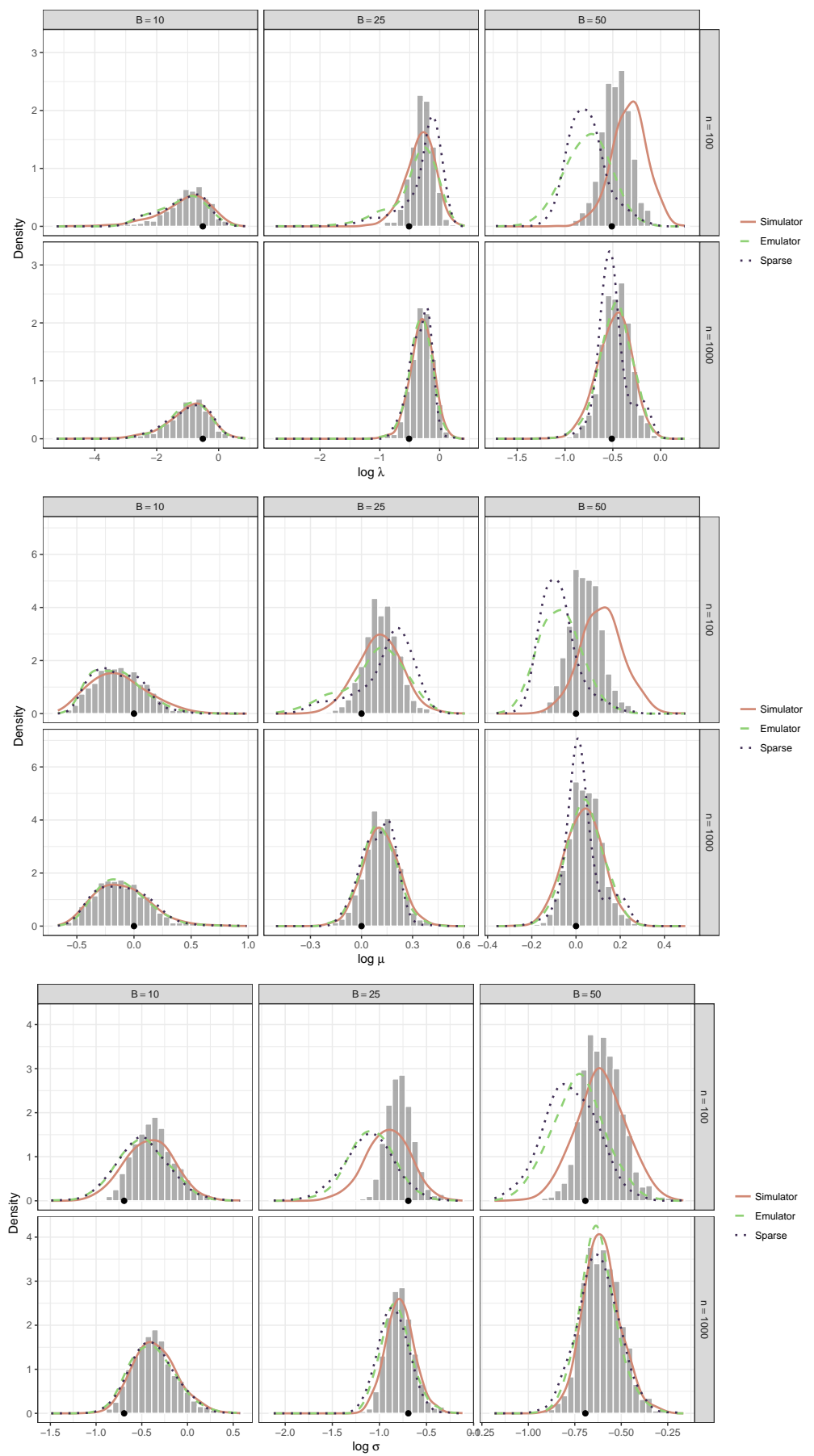

Figure 2: Marginal posterior density histograms for $\lambda$ (top), $\mu$ (middle) and $\sigma$ (bottom) using the exact inference scheme on noise-free data, together with posterior densities obtained using the simulator and non-sparse and sparse emulators on noisy data. Rows refer to the number of replicates used in the simulator $(n)$ and columns refer to the level of discretisation on the data $(B)$. The true values are given by solid circles. 
space and that the true parameter values are recovered well in all cases. In particular, there is very little difference in the marginal posterior distributions when the proportions are calculated using $n=1000$ realisations. Unsurprisingly, the (marginal) posterior distributions obtained using the 'exact' scheme are the most precise, and those obtained by using one of the approximate methods are fairly similar. Indeed for $n=1000$, the emulators produce marginal posterior distributions that are almost indistinguishable from those produced using the simulator, with those for $n=100$ suffering from only a slight loss of precision.

\subsection{Emulator diagnostics}

Although we have seen that using emulators to determine the posterior distribution in this simple birth-death model gives pretty accurate results, in general it is good practice to check whether there are any obvious discrepancies between the underlying sampling distribution of the stochastic process at a particular time and that produced by the emulator. There are a variety of diagnostic tools available in the literature; see, for example, Bastos and O'Hagan [2009]. Most of these are out-ofsample diagnostics and make use of a further set of training data $\mathcal{D}_{t}^{\dagger}=\left\{\left(\boldsymbol{\theta}_{i}^{\dagger}, x_{t, n}^{\dagger}\left(\boldsymbol{\theta}_{i}^{\dagger}\right)\right), i=1, \ldots, n_{d}^{\dagger}\right\}$

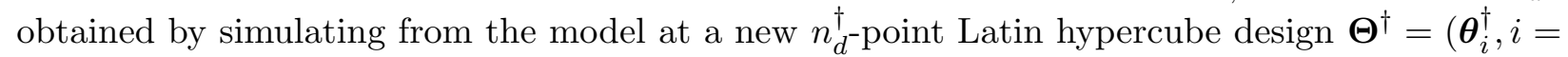
$\left.1, \ldots, n_{d}^{\dagger}\right)$. One diagnostic calculates individual prediction errors (IPE) at each point in the LHD as $d_{t}\left(\boldsymbol{\theta}_{i}^{\dagger}\right)=\left\{x_{t, n}^{\dagger}-m_{t}^{*}\left(\boldsymbol{\theta}_{i}^{\dagger}\right)\right\} / \sqrt{ } \tilde{K}_{t}\left(\boldsymbol{\theta}_{i}^{\dagger}, \boldsymbol{\theta}_{i}^{\dagger}\right)$. Graphical summaries of the IPEs can be useful to assess emulator performance. For example, if the emulator is fitting correctly then the distribution of the IPEs should be standard normal. Large negative or positive IPEs indicate that the emulator variance has been underestimated. Conversely, too many very small values indicate that the emulator variance is inflated. An alternative way of assessing the IPEs is to modify them using the probability integral transform [PIT, Gneiting et al., 2007]. The underpinning assumptions of the GP require that the $d\left(\boldsymbol{\theta}_{i}^{\dagger}\right)$ should follow a standard normal distribution. Therefore PIT statistics $\Phi\left\{d\left(\boldsymbol{\theta}_{i}^{\dagger}\right)\right\}$ should follow a standard uniform distribution. It has been suggested that plots of these PIT statistics allow departures from the GP's distributional assumptions to be detected more easily. Finally, an omnibus measure of the overall fit which also accounts for the correlation between outputs can be determined by calculating a Mahalanobis distance, here given by

$$
M D_{t}^{2}\left(\boldsymbol{\Theta}^{\dagger}\right)=\left\{\boldsymbol{x}_{t, n}-\boldsymbol{m}_{t}^{*}\left(\boldsymbol{\Theta}^{\dagger}\right)\right\}^{\top} \tilde{K}\left(\boldsymbol{\Theta}^{\dagger}, \boldsymbol{\Theta}^{\dagger}\right)^{-1}\left\{\boldsymbol{x}_{t, n}-\boldsymbol{m}_{t}^{*}\left(\boldsymbol{\Theta}^{\dagger}\right)\right\}
$$

If the GP assumption is plausible then, given the GP parameters, whether the emulator provides a good fit can be assessed by comparing its value with a $\chi^{2}$-distribution with $n_{d}^{\dagger}$ degrees of freedom.

Figure 3 shows the GP diagnostics for each of the 10 non-sparse emulators (i.e. $B=10$ ) fitted to training data proportions (at $n_{d}^{\dagger}=75$ points), where each proportion has been calculated using $n=1000$ simulator realisations. It contains IPEs with central $95 \%$ of standard normal distribution, PIT statistics and Mahalanobis distance with central 95\% confidence interval for a $\chi^{2}$ distribution with $n_{d}^{\dagger}=75$ degrees of freedom. The equivalent set of diagnostic plots for the sparse emulator are shown in Figure 4. Both figures are representative of the diagnostics for the other emulators (with different $n$ and $B$ ). Overall the emulators appear to fit fairly well and there are no unusually large IPE values. There is some evidence of a lack of fit for some emulators, with deviation from uniformity in the PIT histograms and the occasional slightly large Mahalanobis distance. The diagnostics for the sparse emulators suggest that their fit is similar to the non-sparse emulators, though the fit may get worse if the sparsity level were to be increased to say $95 \%$. 

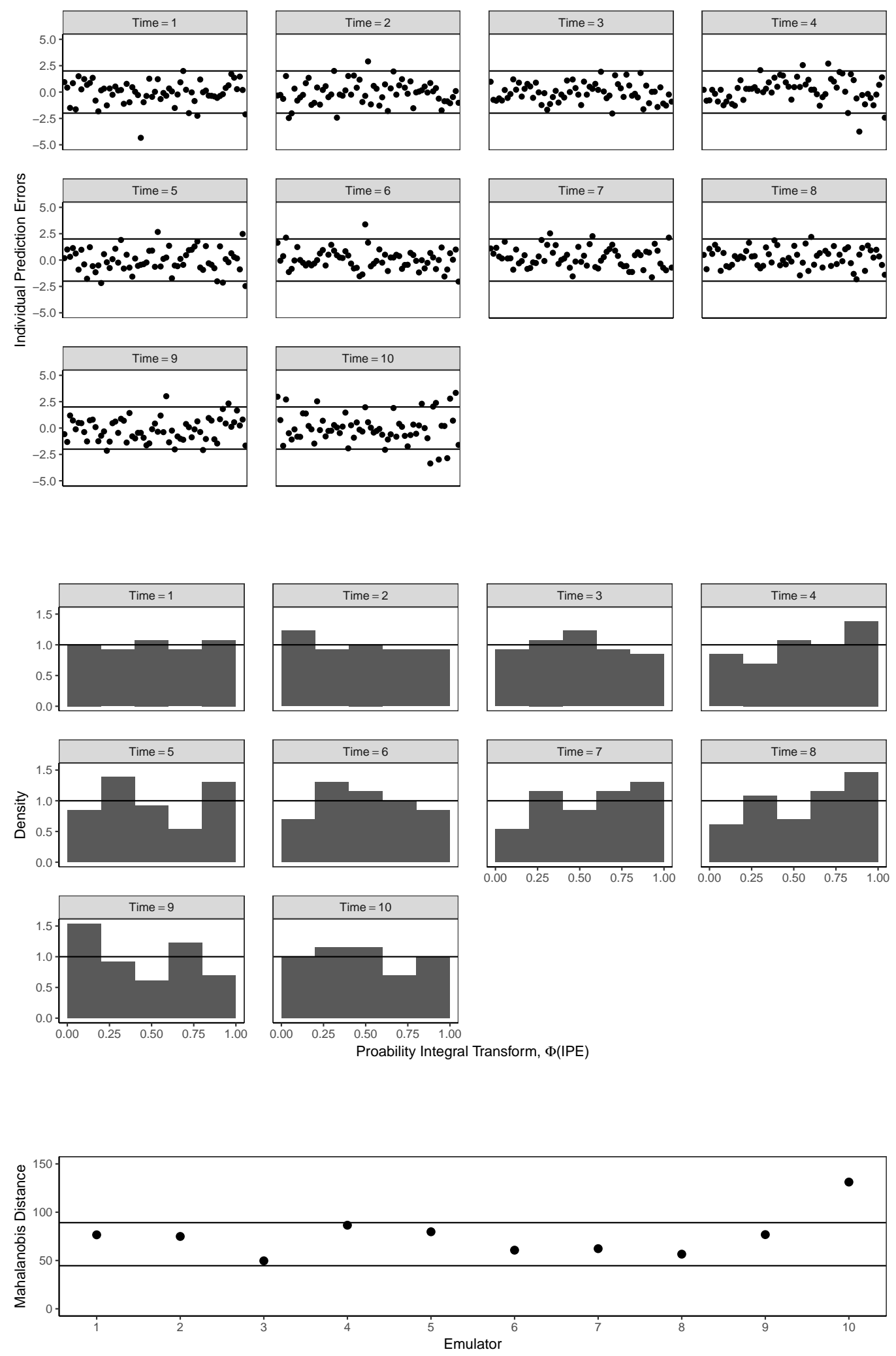

Figure 3: Diagnostics for 10 non-sparse emulators (i.e. $B=10$ ), fitted to training data generated from a simulator with $n=1000$. Individual prediction errors with central $95 \%$ of standard normal distribution indicated (top), probability integral transform (middle) and Mahalanobis distance with central $95 \%$ confidence interval of $\chi_{75}^{2}$ indicated (bottom). 

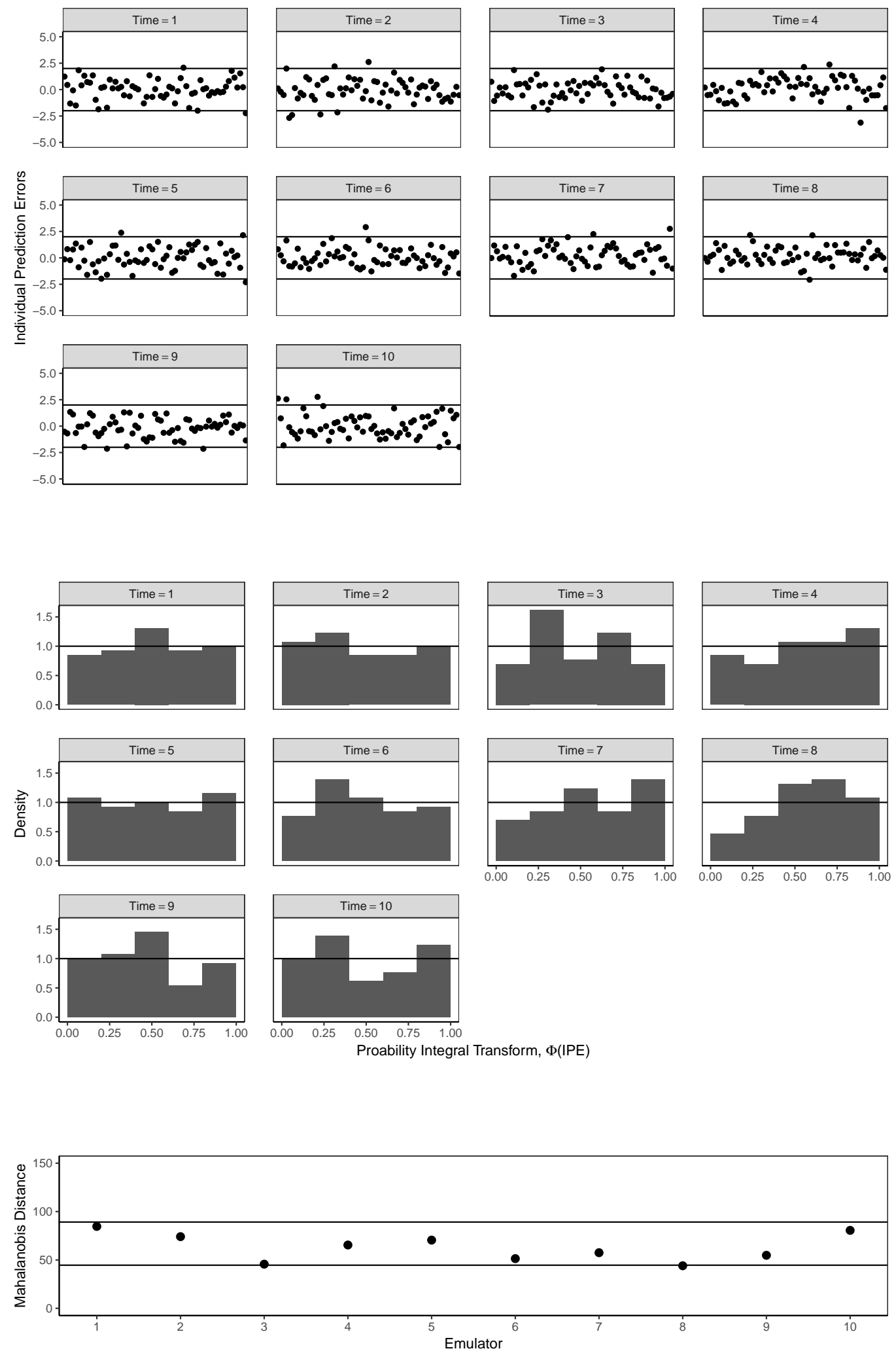

Figure 4: Diagnostics for 10 sparse emulators (i.e. $B=10$ ), fitted to training data generated from a simulator with $n=1000$. Individual prediction errors with central $95 \%$ of standard normal distribution indicated (top), probability integral transform (middle) and Mahalanobis distance with central $95 \%$ confidence interval of $\chi_{75}^{2}$ indicated (bottom). 


\section{Conclusion}

We have examined the effect of various data-poor scenarios on the accuracy of posterior inferences for the parameters of a birth-death process. This simple process is used to describe the time evolution of the alive-status of individual cells. Cell death is assumed to occur when its internal population (as described by the birth-death process) becomes extinct. We have focused our attention on the implications of only being able to measure the proportions of dead cells at certain times rather than actual counts on the underlying cell population.

We first considered the scenario where the probability of cell death was available as an analytic expression, and considered three data-poor scenarios. Typically analytic expressions are not available in realistic models but, as it is available for the birth-death process, this gives us a benchmark posterior distribution against which to compare those obtained via simulation-based approaches. We constructed inference schemes for these data-poor scenarios and noted that observing discretised death times rather than exact death times has little effect on the posterior distribution. However, observing exact proportions of cell death (even with a modest level of noise) leads to more precise (marginal) posterior distributions.

We then considered the more realistic scenario in which an analytic expression is not available for the probability of cell death. Instead we constructed inference schemes based on estimates of such probabilities obtained by simulating many trajectories from the underlying stochastic model. Although simulating these trajectories for the simple birth-death process is very fast, this is not the case for models of reasonable size and complexity, and so alternative strategies are needed. We developed an inference scheme based on a Gaussian process approximation (emulator) to the simulator. We also investigate any further computational gains that might be found by taking advantage of the near sparsity of the emulator's covariance function.

Comparing the various approximate (marginal) posterior distributions with those obtained using the analytic expression for the probability of cell death shows that the approximate methods all produce reasonably accurate posteriors. In all cases, the (approximate) posterior distribution is located in very similar regions of parameter space and the true parameter values are recovered well. In particular, there is very little difference in the marginal posterior distributions when the proportions are calculated using $n=1000$ realisations.

We conclude with a general discussion of when, within the scenarios we discuss in this paper, Gaussian process approximations can be usefully employed in inference algorithms. GP-based inference algorithms can be much more efficient if the time taken to fit them is relatively quick compared to generating realisations using a slow simulator. In this paper the underlying model of cellular death is governed by the birth-death model and as realisatons from this model are very quick to simulate, it is never more efficient to use a GP-based algorithm. However, with more complex models which take longer to simulate, considerable gains can be found by employing GP approximations. For example, in our simulation-based algorithms we need to simulate $n$ model realisations over a time interval $(0, T)$ to obtain each proportion $\widehat{p}_{t, n}$. Suppose this typically takes $n \tau T$ cpu units, where $\tau T$ is the time to simulate a $(0, T)$ realisation. Then running the inference algorithm using the simulator (as described in section 4) for $N_{i t e r}$ iterations takes $n \tau T N_{i t e r}$ cpu units. In contrast, generating the proportions at all of the $n_{d}$ training points (needed to train the GPs) will take $n_{d} n \tau T \mathrm{cpu}$ units. The main computational expense of fitting a GP to the proportions (at a particular time-point) is in inverting an $n_{d} \times n_{d}$ matrix at each iteration of the MCMC fitting algorithm. This task is typically $\mathcal{O}\left(n_{d}^{3}\right)$, and so using $N_{i t e r}^{G P}$ iterations to fit GPs at all $T$ time-points will take roughly $T n_{d}^{3} N_{i t e r}^{G P}$ cpu units. Finally, running the inference algorithm using the $T$ fitted GPs (as described in section 4.1) for $N_{i t e r}^{G P f i t}$ iterations will take around $T n_{d}^{3} N_{i t e r}^{G P f i t}$

cpu units. Thus in total the GP-based inference algorithm will take $T n_{d}\left\{n \tau+n_{d}^{2}\left(N_{\text {iter }}^{G P}+N_{\text {iter }}^{G P \text { fit }}\right)\right\}$ cpu units. Further gains can be found by employing sparse GP approximations (as described in 
section 4.1.1), though Kaufman et al. [2011] do not provide any measure of improvement that depends on the sparsity level $s$. We have found that the number of iterations needed in each inference algorithm is very similar - this shouldn't be surprising as each simulator/emulator is approximating the same sampling distribution at each time-point - and so $N_{i t e r} \simeq N_{i t e r}^{\text {GPfit }}$ for both GP-based algorithms. In conclusion, using these rough scalings, a GP-based algorithm will be more efficient if $n_{d}^{3}\left(N_{i t e r}^{G P}+N_{\text {iter }}\right)<n \tau\left(N_{\text {iter }}-n_{d}\right)$ and, of course, this will be true when the time $\tau T$ to simulate a $(0, T)$ realisation is reasonably large.

\section{References}

Christophe Andrieu, Arnaud Doucet, and Roman Holenstein. Particle Markov chain Monte Carlo methods (with discussion). Journal of the Royal Statistical Society: Series B (Statistical Methodology), 72(3):269-342, 2010. ISSN 13697412. URL http://dx.doi.org/10.1111/j. $1467-9868.2009 .00736 . x$.

Andrew W. Baggaley, Richard J. Boys, Andrew Golightly, Graeme R. Sarson, and Anvar Shukurov. Inference for population dynamics in the neolithic period. Annals of Applied Statistics, 6(4): 1352-1376, 12 2012. URL http://dx.doi.org/10.1214/12-A0AS579.

Leonardo S. Bastos and Anthony O'Hagan. Diagnostics for Gaussian process emulators. Technometrics, 51:425-438, November 2009. ISSN 0040-1706. URL http://dx.doi.org/10.1198/TECH. 2009.08019.

Mogens Bladt and Michael Sørensen. Statistical inference for discretely observed markov jump processes. Journal of the Royal Statistical Society. Series B (Statistical Methodology), 67(3): 395-410, 2005. URL http://www. jstor.org/stable/3647667.

R J Boys, D J Wilkinson, and T B L Kirkwood. Bayesian inference for a discretely observed stochastic kinetic model. Statistics and Computing, 18:125-135, 2008. URL http://dx.doi.org/ $10.1007 / \mathrm{s} 11222-007-9043-\mathrm{x}$.

Forrest W. Crawford and Marc A. Suchard. Transition probabilities for general birth-death processes with applications in ecology, genetics, and evolution. Journal of Mathematical Biology, 65(3): 553-580, 2012. ISSN 0303-6812. URL http://dx.doi.org/10.1007/s00285-011-0471-z.

Forrest W. Crawford, Vladimir N. Minin, and Marc A. Suchard. Estimation for general birthdeath processes. Journal of the American Statistical Association, 109(506):730-747, 2014. ISSN 0162-1459. URL http://dx.doi.org/10.1080/01621459.2013.866565.

Dominique Dehay and Jian-Feng Yao. On likelihood estimation for discretely observed Markov jump processes. Australian 83 New Zealand Journal of Statistics, 49(1):93-107, 2007. ISSN 13691473. URL http://dx.doi.org/10.1111/j.1467-842X.2006.00466.x.

W Feller. Die Grundlagen der Volterraschen Theorie des Kampfes ums Dasein in wahrscheinlichkeitstheoretischer Behandlung. Acta Biotheoretica, 5:11-40, 1939.

G. J. Gibson and E. Renshaw. Estimating parameters in stochastic compartmental models using Markov chain methods. Mathematical Medicine and Biology, 15(1):19-40, 1998. ISSN 1477-8599. URL http://dx.doi.org/10.1093/imammb/15.1.19.

G. J. Gibson and E. Renshaw. Inference for immigration-death processes with single and paired immigrants. Inverse Problems, 17(3):455-466, 2001. ISSN 0266-5611. URL http://dx.doi.org/ $10.1088 / 0266-5611 / 17 / 3 / 306$. 
Colin S Gillespie and Eric Renshaw. The evolution of a single-paired immigration death process. Journal of Physics A: Mathematical and Theoretical, 41(35):355002, 2008. URL http://stacks . iop.org/1751-8121/41/i=35/a=355002.

Tilmann Gneiting, Fadoua Balabdaoui, and Adrian E. Raftery. Probabilistic forecasts, calibration and sharpness. Journal of the Royal Statistical Society, Series B, 69:243-268, April 2007. ISSN 1369-7412. URL http://doi.wiley.com/10.1111/j.1467-9868.2007.00587.x.

A Golightly and D J Wilkinson. Bayesian inference for stochastic kinetic models using a diffusion approximation. Biometrics, 61(3):781-788, 2005. URL http://www. jstor.org/stable/3695607.

D. A. Henderson, R. J. Boys, K. J. Krishnan, C. Lawless, and D. J. Wilkinson. Bayesian emulation and calibration of a stochastic computer model of mitochondrial DNA deletions in substantia nigra neurons. Journal of the American Statistical Association, 104(485):76-87, 2009. URL http://dx.doi.org/10.1198/jasa.2009.0005.

D A Henderson, R J Boys, and D J Wilkinson. Bayesian calibration of a stochastic kinetic computer model using multiple data sources. Biometrics, 66(1):249-256, 2010. ISSN 1541-0420. doi: 10.1111/ j.1541-0420.2009.01245.x. URL http://dx.doi.org/10.1111/j.1541-0420.2009.01245.x.

Cari G. Kaufman, Derek Bingham, Salman Habib, Katrin Heitmann, and Joshua A. Frieman. Efficient emulators of computer experiments using compactly supported correlation functions, with an application to cosmology. Annals of Applied Statistics, 5(4):2470-2492, December 2011. ISSN 1941-7330. URL http://projecteuclid.org/euclid.aoas/1324399603.

D G Kendall. On the generalized 'birth-and-death' process. Annals of Mathematical Statistics, 19: 1-15, 1948. doi: 10.1214/aoms/1177730285.

Marc C. Kennedy and Anthony O'Hagan. Predicting the output from a complex computer code when fast approximations are available. Biometrika, 87(1):1-13, 2000. URL http://dx.doi.org/ 10.1093/biomet/87.1.1.

Marc C. Kennedy and Anthony O'Hagan. Bayesian calibration of computer models. Journal of the Royal Statistical Society: Series B (Statistical Methodology), 63(3):425-464, 2001. URL http://dx.doi.org/10.1111/1467-9868.00294.

J. Owen, Darren J. Wilkinson, and Colin S. Gillespie. Likelihood free inference for Markov processes: a comparison. Statistical Applications in Genetics and Molecular Biology, 14:189-209, 2015. URL https://doi.org/10.1515/sagmb-2014-0072.

Carl Edward Rasmussen and Christopher K. I Williams. Gaussian processes for machine learning. The MIT Press, 2006. ISBN 0-262-18253-X. URL http://citeseerx.ist.psu.edu/viewdoc/ summary?doi=10.1.1.86.3414.

E Renshaw. Modelling Biological Populations in Space and Time (Cambridge Studies in Mathematical Biology). Cambridge: Cambridge University Press, 1993. ISBN 0521448557.

Amar Shah, Andrew Wilson, and Zoubin Ghahramani. Student-t processes as alternatives to Gaussian processes. In Samuel Kaski and Jukka Corander, editors, Proceedings of the Seventeenth International Conference on Artificial Intelligence and Statistics, volume 33 of Proceedings of Machine Learning Research, pages 877-885, Reykjavik, Iceland, 22-25 Apr 2014. Proceedings of Machine Learning Research. URL http://proceedings.mlr.press/v33/shah14.html. 
G Udny Yule. A mathematical theory of evolution, based on the conclusions of Dr. J. C. Willis, F.R.S. Philosophical Transactions of the Royal Society of London, Series B, Containing Papers of a Biological Character, 213(402-410):21-87, January 1925. URL http://dx.doi.org/10.1098/ rstb.1925.0002. 